\title{
Simulation and Reverse Tasks in Macromolecular Reactions and Structure of Polymeric Liquid Crystals
}

\author{
Dedicated to the Memory of the late Professor Ichiro Sakurada \\ N. A. Platé, L. B. Stroganov, and O. V. Noah \\ Polymer Chemistry Department, Moscow State University, \\ Moscow, USSR
}

(Received February 9, 1987)

\begin{abstract}
Simulation of macromolecular reactions (intramolecular crosslinking and hydrolysis of poly(vinyl acetate)) having conformational effect was carried out, and the experimental data and the calculated data based on Monte-Carlo procedure were compared. The situation with rate constants data on polymer-analogous reactions described in the literature was critically reviewed by taking into account the ambiguity of the quantitative conclusions made, and a mathematical procedure to find correct data is proposed. The reverse tasks in polymer physics were analyzed on the example of the ${ }^{1} \mathrm{H}$ NMR spectroscopy of polymeric thermotropic liquid crystals, and an original approach to derive valuable information on the orientational structure in a smectic polymer from its ${ }^{1} \mathrm{H}$ NMR spectrum is proposed.

KEY WORDS Macromolecular Reactions / Monte-Carlo Procedure / Intramolecular Crosslinking / Simulation / Polymeric Liquid Crystal / ${ }^{1} \mathrm{H}$ NMR Spectroscopy / Orientation / Smectic / Second Moment / Resolution of Spectra /
\end{abstract}

The field of simulation and reverse tasks in polymer science is now very wide and cannot be reviewed in a short paper. Therefore, the aim of the present authors is to demonstrate, on some examples from their own studies, the problems, methods of their solutions and some results characteristic for this field.

\section{SIMULATION}

Since even an isolated macromolecule is a complicated statistical ensemble of small fragments or units, simulation is a very useful method of the study of macromolecular processes. One such process is a macromolecular reaction, the features of which are related with the neighboring group effects, and configurational, conformational, supermolecular and other effects. ${ }^{1,2}$ The field of polymer-analogous reactions is a branch of macromolecular chemistry developed by the classical works of I. Sakurada on the hydrolysis of poly(vinyl acetate $)^{3,4}$ for which the rate constants for the syndio- and isotactic polymers were first evaluated and the first reliable conclusion about the role of the configurational effects in such processes was made.

During past years, the simulation was successfully applied to the study of the conformational effect in macromolecular reactions. ${ }^{5-8}$ The role of the conformational effect is very essential for intramolecular crosslinking of a macromolecule where the interaction between two functional groups separated by tens of units is possible only if they come close to each other in a particular conformation of a chain. On the other hand, the reactions of this type are accompanied by a change of chain conformation due to the chain coiling with the accumulation of crosslinks.

Analytical calculation of the kinetics of crosslinking and equilibrium properties of par- 

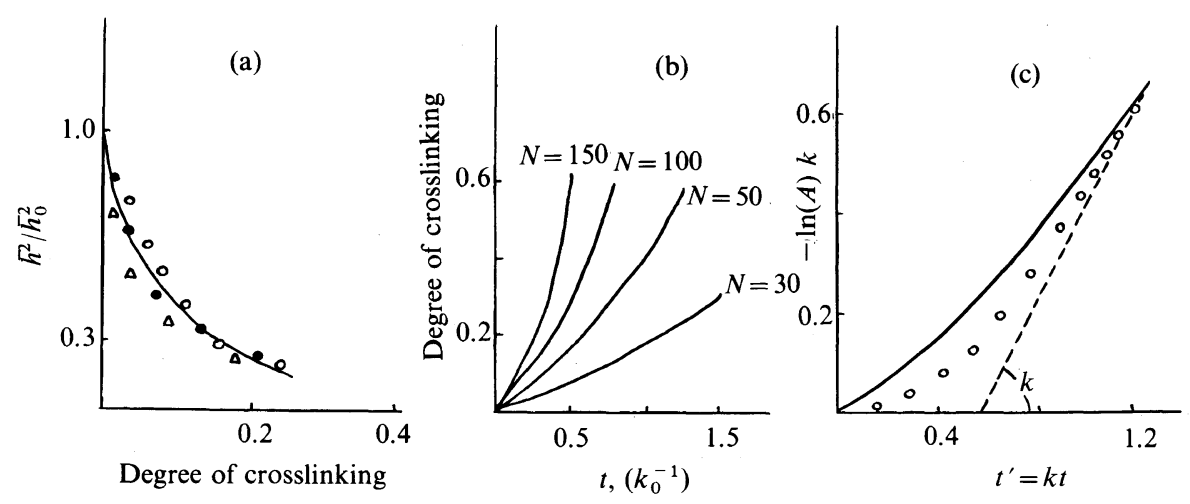

Figure 1. Simulation of intramolecular crosslinking.

tially crosslinked coils is a problem difficult to solve because of the rapid increase of the number of possible topological structures and the essential complication of the calculation algorithm for each of them. However, the Monte-Carlo method, successfully applied by us for this case,$^{5-7}$ permits evaluation of some important parameters. The average dimensions of macromolecular coils decrease with the degree of crosslinking, and this effect is more pronounced for a longer chain. In Figure 1a, the calculated data on the change of $\bar{h}^{2} / \bar{h}_{0}^{2}$ are compared with the experimental ones obtained for $\operatorname{poly}(\mathrm{N}$-methacryloyl-L-lisine) intramolecularly crosslinked with $\mathrm{Cu}(\mathrm{II})$ ions. ${ }^{9}$ The agreement between them is rather satisfactory.

The kinetic curves obtained by computation (Figure 1b) indicate the existence of an autoacceleration effect accompanying the crosslinking reaction.

Another important problem is the quantitative account of the conformational effect in polymer-analogous reactions. In this case, the conformational effect is demonstrated by the change of the reactivity of functional groups of a macromolecule as a result of the change of their accessibility to a low-molecular-weight reagent after the transition from one conformation to another.

An example of a reaction proceeding with such an effect is the alkaline hydrolysis of PVA in an acetone-water mixed solvent. ${ }^{10}$ The ki- netic curve of this reaction is characterized by the existence of an induction time up to $20 \%$ conversion; above this conversion, the kinetics is described by an equation of the second order with the rate constant close to that for the lowmolecular analogs. Such kinetic behavior can be explained by the gradual unfolding of a coil due to the change of the solvent quality, in the course of $\mathrm{OH}$ group accumulation, from poor for PVA to good for the copolymer with $20 \%$ of alcohol groups. Beyond $20 \%$ conversion, all the acetate groups are equally accessible to the hydrolyzing agent.

The reversible folding-unfolding process of a macromolecular coil related with the change of the solvent quality can also be simulated for reversible crosslinking-"uncrosslinking" accompanied by analogous conformational changes. ${ }^{8}$ Accepting the "pseudocrosslinking" hypothesis, we do not assume here the formation of any real physical bonds, but only the possibility of application of mathematics to intramolecular crosslinking reactions to describe the conformational effect in the polymer-analogous reaction.

Parameters of the simulation procedure for the reversible intramolecular "pseudocrosslinking" can be determined for each particular reaction by independent experiments. The main parameter is the equilibrium constant $(K)$ of the reversible "pseudocrosslinking" which can be expressed by $\Delta F_{\text {mix }}$ for any 
reversible process:

$$
\begin{gathered}
k T \ln K=-\partial \Delta F_{\text {mix }} / \partial n_{2} X \\
\Delta F_{\text {mix }}=\Delta H_{\text {mix }}+T \Delta S_{\text {mix }}
\end{gathered}
$$

It follows from the lattice model that

$$
\Delta F_{\text {mix }}=\Delta H_{\text {mix }}+k T\left(n_{1} \ln v_{1}+n_{2} \ln v_{2}\right)
$$

where $n_{1}$ and $n_{2}$ are the numbers of solvent and polymer molecules, respectively, $v_{1}$ and $v_{2}$ are the solvent and polymer volume fractions, and $X$ is the degree of polymerization.

In the framework of the proposed model, the conformational effect is manifested in the change of $K$ in the course of the reaction. This change can be followed by measuring $\Delta H_{\text {mix }}$ for copolymers that are the intermediate products of the reaction.

Figure 1c shows the kinetic curve for the polymer-analogous reaction obtained by computer simulation under the condition that competing intramolecular "pseudocrosslinking" has an equilibrium constant determined by the thermodynamic method. In the same figure, the experimental data for the kinetics of PVA hydrolysis are presented. It is seen that the computational curve satisfactorily describes the experimental data.

This last example demonstrates the possibility that the computer simulation method provides new quantitative information about such well-studied reactions as PVA hydrolysis, the kinetic constants of which were firstly calculated in Sakurada's works 20 years ago. ${ }^{4}$

\section{SOLUTION OF REVERSE TASKS}

The simulation of macromolecular reactions or the solution of direct tasks of calculation is an important area of polymer science, but the correct organization of an experiment itself and evaluation of the model parameters, i.e., the solution of the reverse tasks, is of the same importance.

\section{REVERSE TASKS AND NEIGHBORING GROUP EFFECTS}

During the last fifteen years, success was achieved essentially in the solution of direct tasks of the theory of neighboring group effects in polymer-analogous reactions. The achievement includes the calculation of kinetics, units sequence distribution and compositional distribution functions for intermediate copolymer products from the rate constants $k_{0}, k_{1}$, and $k_{2}$ of the corresponding reactions of functional groups having none, one and two reacted nearest neighbors, respectively. The problem is solved by the integration of complicated systems of differential equations. ${ }^{1,2,11}$

The theory permits the evaluation which is very important for polymer science, but further progress in this field is impossible without the knowledge of numerical values of constants. Therefore, the experimental determination of these values is one of the most important tasks of modern macromolecular chemistry.

The information about the individual rate constants can be obtained from experimental data of four types: 1) kinetic data, 2) data about the compositional distribution functions, 3) NMR data on the change of triads concentrations with time, and 4) analogous data on the dependence of triads concentrations on the copolymer composition. There are thus four particular problems; each of them includes two ones because the initial product can be a homo- or copolymer. Finally, for each problem two types of programs should be worked out. The first etalonic method providing the most accurate solution of the task is oriented toward the use of a large high-speed computer. These programs are rather complicated, require personnel having high qualification, and should serve for the study of the main methodical questions and determination of the limits of the applicability of other approximate methods oriented to a 

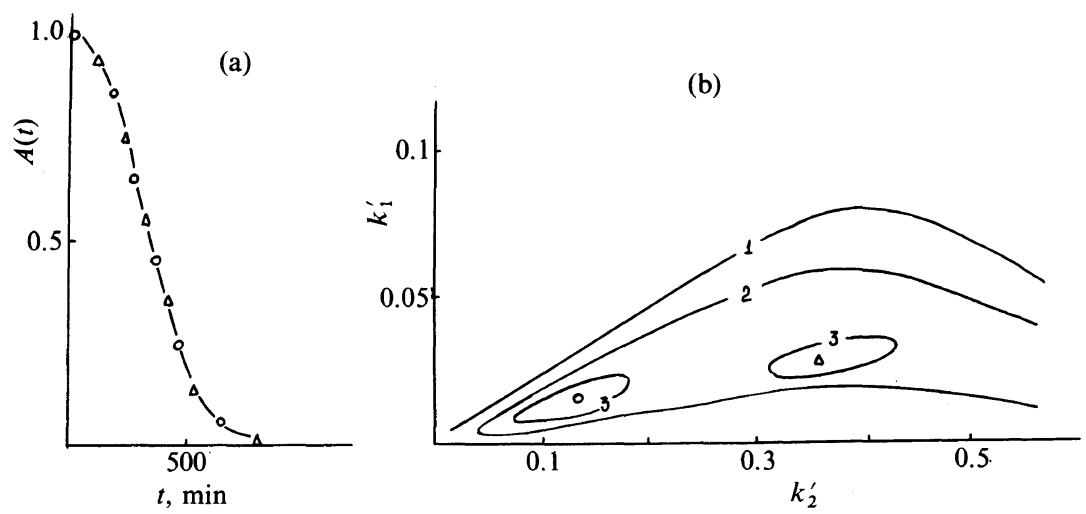

Figure 2. Kinetics of the polymer-analogous reaction (a) and topography of the surface of the sum of deviation squares $S\left(k_{1}^{\prime}, k_{2}^{\prime}\right)$ in the vicinity of minima (b); $\bigcirc, k_{0}: k_{1}: k_{2}=1: 10: 100, S=0 ; \triangle, k_{0}: k_{1}: k_{2}$ $=1: 11: 17, S=10^{-6} .1, S=5 \times 10^{-4} ; 2, S=10^{-4} ; 3, S=5 \times 10^{-6}$.

mini-computer. The latters, second type of programs, could serve for immediate practical usage. Thus, the program pack worked out by us includes 16 particular reverse tasks of the theory of the neighboring groups effects.

All the programs are built following the classical scheme including the minimization of the sum of squares of deviations of experimental and theoretical data $(S)$. It is known $n^{1,2,11}$ that the problem of $S$ minimization for such models is rather complicated because the three-dimensional $S$ surface of the rate constants can have several minima. So, in Figure 2a, accurate kinetic curves are presented which were calculated by a computer for the rate constants ratios $k_{0}: k_{1}: k_{2}=1: 10: 100$ and $1: 11: 17$. The curves agree with an accuracy higher than $10^{-5}$, while the accuracy of the kinetic experiment itself is usually lower than $10^{-2}$; i.e., an ambiguity appears in the evaluation of the constants. We have studied the mutual disposition of minima pairs using a specially worked-out formalism for the transition to the two-dimensional surface ${ }^{12}$ and computer graphics. In Figure $2 b$, a part of the $S$ surface in the two-dimensional presentation for the case mentioned above is shown. The minima precisely correspond to the constant values given in Figure $2 a$. A study of many such topographic maps has led to empirical relationships permitting a transition from the coordinates of one minimum to a vicinity of another. Based on this relationship, the algorithm has been derived which enables one, after the determination of one constant set by the minimization program, to calculate a new initial approximation in the vicinity of another minimum and, with the aid of the second minimization, and to find the evaluations of a second set of rate constants.

Another difficulty is related to the ravine character of the squares sum surface. Usual minimization algorithms are ineffective for this. Therefore we have worked out a special algorithm ${ }^{13}$ adapted to the changed ravine surface structure.

For practical reasons, it is very important to foresee conditions (conversion, experimental error, etc.) which permit the unambiguous evaluation of constants. To find the unambiguity region, we checked, using the Fisher criterion, the hypothesis for the statistical negligibility of the difference between the minimum height and the experimental accuracy level. The limits of the unambiguity region for the experimental accuracy, 1 and $2 \%$, are presented in Figure 3. One can see that even for such high experimental accuracy, most of the possible rate constants belong to the ambiguity region. 


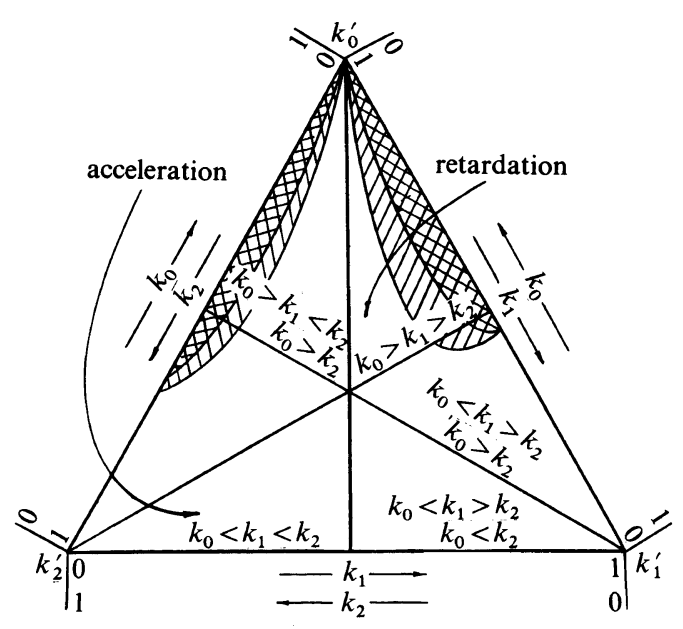

Figure 3. Ambiguity of rate constant evaluation: the unambiguity region for experimental accuracy equal to $1 \%$; the unambiguity region for experimental accuracy equal to $2 \%$.

In Table I, the results of various studies of the kinetics of polymer-analogous reactions are summarized. The rate constants that were published without taking into account the ambiguity are supplemented by our evaluation of the rate constants of the same reactions. It is obvious that in many cases, two constant sets correspond to quite different chemical implications of neighboring group effects. Unfortunately, the unambiguity problem cannot be solved without all initial experimental information, but the main conclusion is evident. The correct analysis of such complicated kinetic models requires to take into account the possibility of the existence of several constant sets describing the same experimental data.

Unlike the kinetic data, the extraction of information on constants from the data on the triads of units leads to an unambiguous result.

Besides the etalonic method for large computer, programs for a mini-computer have been made by taking the Markov chain approximation $^{1}$ as a solution of the direct task and the coordinate descent method as a minimization algorithm. The results of such a calculation almost completely agree with those by the etalonic method. So the programs have been worked out for almost all real problems in this field besides the reverse problems of compositional heterogeneity. Since the experimental data on compositional distribution functions are hardly available and have the low accuracy, the active use of these data for the evaluation of rate constants in the near future is doubtful.

\section{ANALYSIS OF POORLY RESOLVED SPECTRA}

When discussing the reverse tasks, we believe that the experimental data should correspond to the values described by the equations for a model system. But often, the experimental methods give dependences when the extraction of information from them is also related with solutions of complicated reverse problems. Such a situation is very characteristic for polymer science where the standard physico-chemical methods work as a rule within the limits of allowed resolution or sensitivity. Typical examples are poorly resolved spectra consisting of several bell-like components. These are NMR, ESR, IR, and UV spectra, X-ray patterns, chromatograms, luminescence spectra and others. There exist many methods for computer resolution 
Table I. Estimation of constants for reactions with neighboring group effects ${ }^{12}$

\begin{tabular}{|c|c|c|}
\hline Reaction & & \\
\hline $\begin{array}{l}\text { Hydrolysis of PMMA } \mathrm{PM}^{\mathrm{a}}(0.2 \mathrm{M} \mathrm{KOH}) \\
\qquad \begin{array}{l}(0.2 \mathrm{M} \mathrm{KOH}) \\
\left(\mathrm{Py} / \mathrm{H}_{2} \mathrm{O}\right)\end{array}\end{array}$ & $\begin{array}{l}1: 0.4: 0.4 \\
1: 0.2: 0.05 \\
1: 8: 100\end{array}$ & $\begin{array}{l}1: 0.13: 0.5 \\
1: 0.02: 0.3 \\
1: 9: 16\end{array}$ \\
\hline $\begin{array}{r}\text { Hydrolysis of poly(diphenylmethylmethacrylate) iso } \\
\text { syndio }\end{array}$ & $\begin{array}{l}1: 20: 100 \\
1: 1: 1\end{array}$ & $\begin{array}{l}1: 21: 42 \\
1: 0.4: 1.7\end{array}$ \\
\hline Hydrolysis of poly(phenylmethacrylate) & $\begin{array}{l}1:: 40: 1000 \\
1: 55: 100 \\
1: 18: 65 \\
1: 2: 10\end{array}$ & $\begin{array}{l}1: 41: 69 \\
1: 56: 27 \\
1: 18: 45 \\
1: 3: 4\end{array}$ \\
\hline $\begin{array}{c}\text { Chlorination of } \mathrm{PE}^{\mathrm{b}} \quad(1 \text { order }) \\
\text { of } \mathrm{PE} \quad(1 \text { order }) \\
\text { of } \mathrm{PE}(1 / 2 \text { order }) \\
\text { of } n \text {-hexadecane } \\
\text { of cyclo } \mathrm{C}_{8} \mathrm{H}_{15} \mathrm{Cl}, \mathrm{C}_{12} \mathrm{H}_{24}, \mathrm{C}_{28} \mathrm{H}_{56}\end{array}$ & $\begin{array}{l}1: 0.35: 0.08 \\
1: 0.6: 0.36 \\
1: 0.38: 0.11 \\
1: 0.35: 0.08 \\
1: 0.43: 0.18\end{array}$ & $\begin{array}{l}1: 0.03: 0.46 \\
1: 0.04: 0.5 \\
1: 0.03: 0.46 \\
1: 0.06: 0.6\end{array}$ \\
\hline $\begin{array}{l}\text { Quaternization of poly(4-vinylpyridine) } \\
\text { of poly(2-methyl-5-vinylpyridine) }\end{array}$ & $\begin{array}{l}1: 0.3: 0.3 \\
1: 1: 0.1 \\
1: 0.6: 0.1\end{array}$ & $\begin{array}{l}1: 0.1: 0.4 \\
1: 0.03: 1.3 \\
1: 0.03: 0.8\end{array}$ \\
\hline $\begin{array}{l}\text { Epoxidation of polyisoprene }\left(\mathrm{CCl}_{4}\right) \\
\text { (benzene) }\end{array}$ & $\begin{array}{l}1: 0.6: 0.3 \\
1: 0.67: 0.42\end{array}$ & $\begin{array}{l}1: 0.1: 0.8 \\
1: 0.1: 0.9\end{array}$ \\
\hline Reaction of poly(methacryloyl chloride) with amines & $1: 0.1: 0$ & $1: 0: 0.2$ \\
\hline Nucleophylic substitution on PMMA & $1: 0.2: 0$ & $1: 0: 0.03$ \\
\hline Esterification of PMMA & $1: 0.1: 0$ & $1: 0: 0.2$ \\
\hline $\begin{array}{r}\text { Reaction of PMMA with methylsulfonylmethyllithium } \\
\text { iso } \\
\text { syndio }\end{array}$ & $\begin{array}{l}1: 0.055: 0 \\
1: 1: 0.003\end{array}$ & $\begin{array}{l}1: 0: 0.07 \\
1: 0.001: 1.3\end{array}$ \\
\hline
\end{tabular}

a PMMA, poly(methyl methacrylate).

b PE, polyethylene.

of such spectra; in particular, some programs have been proposed by us. ${ }^{14,15}$

The model shape of a line is not analytically calculated as in most analogous programs but is obtained as a result of transfer of an origin of a scale and oblateness of the gauge line shape retained in computer storage. For code programming, such an approach gives more than 1000-fold economy of time and permits creation of cinematographic effect on the visual display. A spectrum consisting of $6-8$ components appears on the visual display with a frequency higher than 20 times per sec., and the human eye perceives this blinking picture as a continuous one. The experimental and model lines are put out simultaneously, and an operator chooses the best model parameters by controlling this "living" spectrum with the aid of special language commands. ${ }^{15}$ The human intuition works in this case much better than the most refined minimization algorithms. Using this method, an operator can find in 5-10 minutes the parameter values in the minimum vicinity, and then the simple 
coordinate descent results in the precise minimum by refining the intensities, widths and positions of line values.

\section{REVERSE TASKS OF ${ }^{1} \mathrm{H}$ NMR \\ OF POLYMERIC LIQUID CRYSTALS}

In past years, a new important area in polymer science has come about, polymeric liquid crystals. This new branch of polymer science greatly needs the analysis of oriented liquid crystalline structures. One of the most perspective methods for the analysis of orientational structures is the wide line ${ }^{1} \mathrm{H}$ NMR spectroscopy, by which the angle dependence of the second moments of spectra of a number of oriented polymers and liquid crystals was determined in the 60's. Later on, however, this method was employed much less frequently because of the small amount and low accuracy of information obtained from the second moments.

Therefore, first of all, we tried ${ }^{19,20}$ to elucidate, for the accurate solution of the reverse task, what is real information about the orientational structure of polymer liquid crystal which can be taken from the angle dependence of the second moment. ${ }^{16,17}$ Rather accurate results can be obtained only for the simplest model that describes the microscopic orientation by the second moment of the spectrum of ideally oriented structure $M$ and corresponds to the representation of the function of orientation distribution of domains $\psi(\theta)$ as a mixture of a monodomain with $\alpha$ contribution<smiles>[CH]CC(=O)OCCOCCOc1ccc(C=Nc2ccc(C#N)cc2)cc1</smiles>

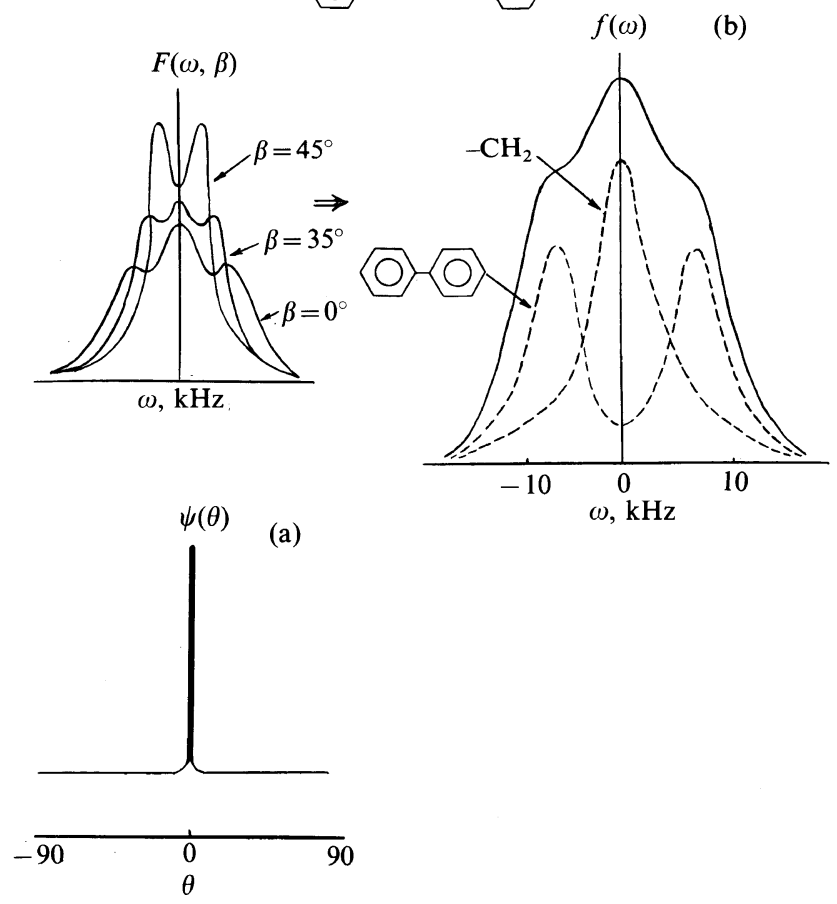

Figure 4. Orientation distribution of structural elements for the T-model (a) and reconstruction of the ${ }^{1} \mathrm{H}$ NMR spectrum of the monodomain structure $f(\omega)$ from the experimental angle dependence of the line shape $F(\omega, \beta)$ of the comb-like polymer smectic (b). - - - spectra corresponding to rigid phenylene and flexible aliphatic fragments. 
and an equally distributed nonoriented domain with $(1-\alpha)$ contribution (Figure $4 a)$. We call this model as "T-model," because the shape of the distribution function resembles an inverse $\mathrm{T}$ letter.

It seems very attractive to push further into this area by increasing the amount of information extracted from an experiment based on measuring the angle dependence of the shape of the line of $F(\omega, \beta)$ spectra and on more informative new methods for solution of the reverse tasks.

We have proposed various computational procedures which are realized in a computer, are studied by the numerical experimental method, and eventually permit, in the framework of the T-model, calculating the complete shape of the ${ }^{1} \mathrm{H}$ NMR spectra corresponding to the ideally oriented monodomain structure $f(\omega)$ and nonoriented part of structural fragments $-\phi(\omega)^{18-20}$ assuming the fast rotation of the oriented fragments around the orientation axis. ${ }^{21}$ We use the property of a spectrum of ideally oriented LC domains ${ }^{21}$, elongated along the intensity axis and compressed along the frequency axis with an increase of the $\beta$ angle with the elongation coefficient $p=$ $2 /\left|\left(3 \cos ^{2}-1\right)\right| .^{18-20}$

$$
F(\omega, \beta)=\phi(\omega)+p f(\omega p)
$$

The differential method includes:

1) Numerical differentiation of the angle dependence $F(\omega, \beta)$ with respect to $p$ to give the angle dependence of the $g(\omega, \beta)$ derivative:

$$
\begin{aligned}
g(\omega, \beta) & =\partial F(\omega, \beta) / \partial p \\
& =f(\omega, p)+\omega p f^{\prime}(\omega p)
\end{aligned}
$$

2) Elongation of $g(\omega, \beta)$ functions with the $1 / p$ coefficient. For an adequate $\mathrm{T}$-model, such elongation should result in the standard $g^{*}(\omega)$ function independent of the $\beta$ angle.

$$
g^{*}(\omega)=f(\omega)+\omega f^{\prime}(\omega)
$$

The comparison of this function calculated for various angles is an internal test of the ade- quacy of the T-model.

3) Averaging the $g^{*}(\omega, \beta)$ values with respect to angles.

4) Numerical integration with respect to the frequency

$$
f(\omega)=\frac{1}{\omega} \int_{0}^{\infty} g^{*}(\omega) \mathrm{d} \omega ; \quad f(0)=g^{*}(0) ; \quad \omega \neq 0
$$

resulting in reconstruction of the ideally oriented structure spectrum $f(\omega)$, while the spectrum of the nonoriented part $\phi(\omega)$ is obtained by subtraction of this reconstructed spectrum from the initial spectra.

Finite Difference Method. To use the differential method, one should have the initial angle dependence rather detailed for numerical differentiation. This disadvantage is absent in the difference method based on a similar idea but representing the function of the nonoriented component shape as its values in the points $\phi(\omega)=\left\{\phi\left(\omega_{1}\right) \cdots \phi\left(\omega_{n}\right)\right\}$ for which the linear equations system for each spectra pair is derived. The shape of the nonoriented component line is reconstructed as a result of the solution of this system, and is further averaged with respect to various angle pairs.

Least Squares Method. Both differential and difference methods need very limited computational resources and are realized by a small computer (ISKRA 226). The disadvantages of both methods are the not very correct averaging of results and the statistical evaluation of errors. The least squares method does not have these disadvantages. This method represents the spectra of nonoriented $\phi(\omega)$ and ideally oriented $f(\omega)$ components by their values in $n$ points and results in a system of $n \cdot m$ conditional equations ( $m$ is the number of angles) with $2 n$ unknowns which are solved by the standard linear variant of the least squares method. For 50 points of a spectrum and 10 angles, there are 500 equations with 100 unknowns. This method is realized by using a large high-speed computer (BESM-6).

All three methods give almost the same 
accuracy close to that of recording the intial spectra, and each method has advantages. The differential method permits checking the adequacy of the T-model. The least squares method gives the best evaluation of parameters and their statistical characteristics. The difference method permits analysis of incomplete angle dependence without an output of information from a small computer controlling the experiment.

In Figure 4b, an example of the calculation of the monodomain component spectrum, in the framework of the T-model, from the experimental angle dependence of ${ }^{1} \mathrm{H}$ NMR spectra of an oriented comb-like smectic polymer is presented. The spectrum of the monodomain fraction has the triplet character which can be explained by the incorporation of some of flexible aliphatic fragments into the orientational structure formed by the rigid phenylene fragments. The computer separation of the spectrum into a narrow central component and a doublet corresponding to the rigid phenylene fragments permits evaluating the fraction of aliphatic fragments incorporated into the orientational structure $(60 \%)$. The distance between the doublet components permits evaluating the order parameter of rigid fragments (0.68).

A comparison of the area corresponding to rigid fragments $(35 \%)$ with their stoichiometric fractions $(37.5 \%)$ permits evaluating average fraction of nonoriented fragments in the LC structure $(6.6 \%)$. Thus, the LC smectic polymer is essentially an oriented polymer. Evidently the reconstructed spectra can be studied as the usual ones obtained by a spectrometer. For example, they can be simulated by quantum mechanical methods based on bond lengths, angles, parameters of mobility of molecular fragments ${ }^{21}$ and so on.

In conclusion of this short review on the field of computer simulation and reverse problems in polymer science, let us outline some general questions concerning the role of reverse problems. Some years ago, the reverse problems seemed to be a mathematical addition to an experiment to elevate its accuracy and to evaluate its errors. However, with development of informational technologies, "the main role" now has gone gradually from an experiment to a reverse task. The structure of a reverse problem affects the choice of experimental conditions. This was shown, for the example, on the ambiguity of evaluation of kinetic constants discussed above. For this case, an investigator is not able to obtain unambiguous conclusions without taking into account the structure of the reverse task when planning an experiment. It seems that already now reverse problems are a very important and intensively developed field of science having its own problems and methods of investigation.

\section{REFERENCES}

1. N. A. Platé, A. D. Litmanovich, and O. V. Noah; "Makromolekulyarnye Reaktsii," Chimiya, Moscow, 1977.

2. N. A. Platé and O. V. Noah, Adv. Polym. Sci., 31, 133 (1979).

3. I. Sakurada, Ann. Rev. Synth. Fiber Soc. Jpn., 1, 192 (1943).

4. I. Sakurada, Pure Appl. Chem., 16, 263 (1968).

5. I. I. Romantsova, Yu. A. Taran, O. V. Noah, and N. A. Platé, Dokl. Akad. Nauk SSSR, 243, 109 (1977).

6. I. I. Romantsova, O. V. Noah, Yu. A. Taran, A. M. Yel'yashevich, Yu. Ya. Gotlib, and N. A. Platé, Vysokomol. Soedin., Ser. A, 19, 2800 (1977).

7. I. I. Romantsova, Yu. A. Taran, O. V. Noah, N. A Platé, Vysokomol. Soedin., Ser. A, 21, 1176 (1979).

8. A. M. Khvan, O. V. Noah, I. D. Zenkov, M. V. Shablygin, and N. A. Platé, Vysokomol. Soedin., Sèr. $A, 28,2251$ (1986).

9. A. M. Khvan, V. V. Chupov, O. V. Noah, and N. A. Platé, Vysokomol. Soedin., Ser. A, 27, 1243 (1985).

10. E. Turska and R. Jantas, J. Polym. Sci., C, N47, 359 (1974).

11. N. A. Platé, O. V. Noah, and L. B. Stroganov, "Proceedings, IUPAC Macromolecules," Pergamon Press, Oxford-New York, 1982, p 65.

12. N. A. Platé, O. V. Noah, and L. B. Stroganov, Vysokomol. Soedin., Ser. A, 25, 2243 (1983).

13. A. N. Olonovskii, L. B. Stroganov, and N. A. Platé, "Novye Pribory, Metodiki, Materialy i Tekhnologicheskie Protsessy, Razrabotannye Uchenymi MGU, Predlagaemye dlya Vnedreniya v Narodnoe 
Khozyaistvo i Nauku," MGU, Moscow, 1982, p 60.

14. L. B. Stroganov, Yu. A. Taran, and N. A. Platé, Vysokomol. Soedin., Ser. A, 17, 2147 (1975).

15. L. B. Stroganov, Yu. A. Taran, and N. A. Platé, $Z h$. Fiz. Khimii, 19, 2696 (1975).

16. Yu. V. Molchanov, A. F. Privalov, Yu. B. Amerik, V. G. Grebneva, and I. I. Konstantinov, Vysokomol. Soedin., Ser. A, 27, 2206 (1985).

17. N. A. Platé, L. B. Stroganov, A. E. Prizment, R. V. Tal'roze, and V. P. Shibaev, Preprints, "International Symposium on Characterization and Analysis of Polymers, Polymer-85," Melbourne, 1985, p 530 .

18. L. B. Stroganov, A. E. Prizment, M. V. Piskunov, A.
N. Olonovskii, and R. V. Tal'roze, "XXII Konferentsiya po Vysokomolekulyarnym Soedineniyam," Alma-Ata, 1985, p 167.

19. N. A. Platé, L. B. Stroganov, and O. V. Noah, "VII Vsesoyuznaya Konferentsiya po Ispol'zovaniyu Vychislitel'nykh Mashin $\mathrm{v}$ Khimicheskikh Issledovaniyakh i Spekroskopii Molekul," Riga, 1986, p 17.

20. L. B. Stroganov, A. N. Olonovskii, and N. A. Platé, "VII Vsesoyuznaya Konferentsiya po Ispol'zovaniiyu Vychislitel'nykh Mashin v Khimicheskikh Issledovaniyakh i Spektroskopii Molekul," Riga, 1986, ì 167.

21. St. Limmer, H. Schmiedel, H. Hillner, A. Lösche, and S. Grande, J. Physique, 41, 869 (1980). 